\title{
Tumor cell plasticity: the challenge to catch a moving target
}

\author{
Sarah Schwitalla
}

Received: 3 February 2014/ Accepted: 4 February 2014/Published online: 25 February 2014

(C) Springer Japan 2014

\begin{abstract}
Every cancer cell is "different"-within one and the same tumor, between different lesions originating from the same tumor, among different patients suffering from the same tumor type, and certainly between different tumor types. The complexity of tumor development, with its genetic, phenotypic and functional heterogeneity and plasticity within tumors and between primary tumors and metastases, underlies the unpredictable influences and stimuli of a tumor-associated inflammatory microenvironment, immune response, mechanical and metabolic stress, therapy-induced inflammation or interaction with microbiota. The stochastic and context dependent nature of these factors accounts for the difficulties to investigate the impact of resulting cell plasticity on tumor development, and justifies the challenge to prevent tumor recurrence. The emerging concept of cell plasticity and reciprocity (to change the phenotype by processing signals from the environment) throws more light on the actual complexity of tumor heterogeneity than can be expected solely from a unidirectional, classical cancer stem cell (CSC) model. To date, it remains widely unclear to what extent cell plasticity impacts tumor development, and it is difficult to assess by current methods. As a high tumor plasticity is likely to predict a poor outcome for patients, the future therapeutic challenge will be the development of personalized treatment strategies to predict and finally prevent cell plasticity in patients.
\end{abstract}

S. Schwitalla $(\square)$

Dr. George Daley Laboratory, Division of Hematology/

Oncology, Boston Children's Hospital, Harvard Stem Cell

Institute, One Blackfan Circle, Boston, MA 02115, USA

e-mail: sarah.schwitalla@childrens.harvard.com
Keywords Tumor cell plasticity - Heterogeneity · CSC model $\cdot$ Stochastic model $\cdot$ Inflammatory microenvironment

\section{Introduction}

Normal tissues display a well-organized tissue structure consisting of different functional cell types residing in their specialized niches. However, in the event of tumor development, the stable cell order, maintained by tissue homeostasis, is replaced by uncontrolled self-renewal of transformed cells, leading to a disorganized tissue structure and the establishment of a new niche, a tumor supportive inflammatory microenvironment, consisting of malignant epithelial cells, abnormal fibroblasts, endothelial cells and diverse infiltrated cytokine secreting immune cells that normally restore the tissue structure under physiologic conditions (Fig. 1). The evolving and expanding genetic and phenotypic tumor tissue heterogeneity confers to the tumor a degenerated structure uncoupled from the homeostatic hierarchical tissue organization. This holds tremendous obstacles for targeting the different tumor cell subpopulations successfully, and results in the event of frequent relapse after treatment, e.g. in colon cancer [1]. The majority of cancer patients die from metastatic dissemination, due to resistance of the tumor towards current therapeutic strategies that are unable to target all phenotypically and genetically diverse tumor cell types. The phenotypic, morphological, signaling, and genetic differences can be observed even between primary and metastatic lesions within the same patient (intrapatient heterogeneity) and within the same lesion (intratumoral heterogeneity) [2,3]. Hence, tumors acquire smart strategies to establish their heterogenous character in order to 
Fig. 1 Inflammation-induced cancer cell plasticity and heterogeneity

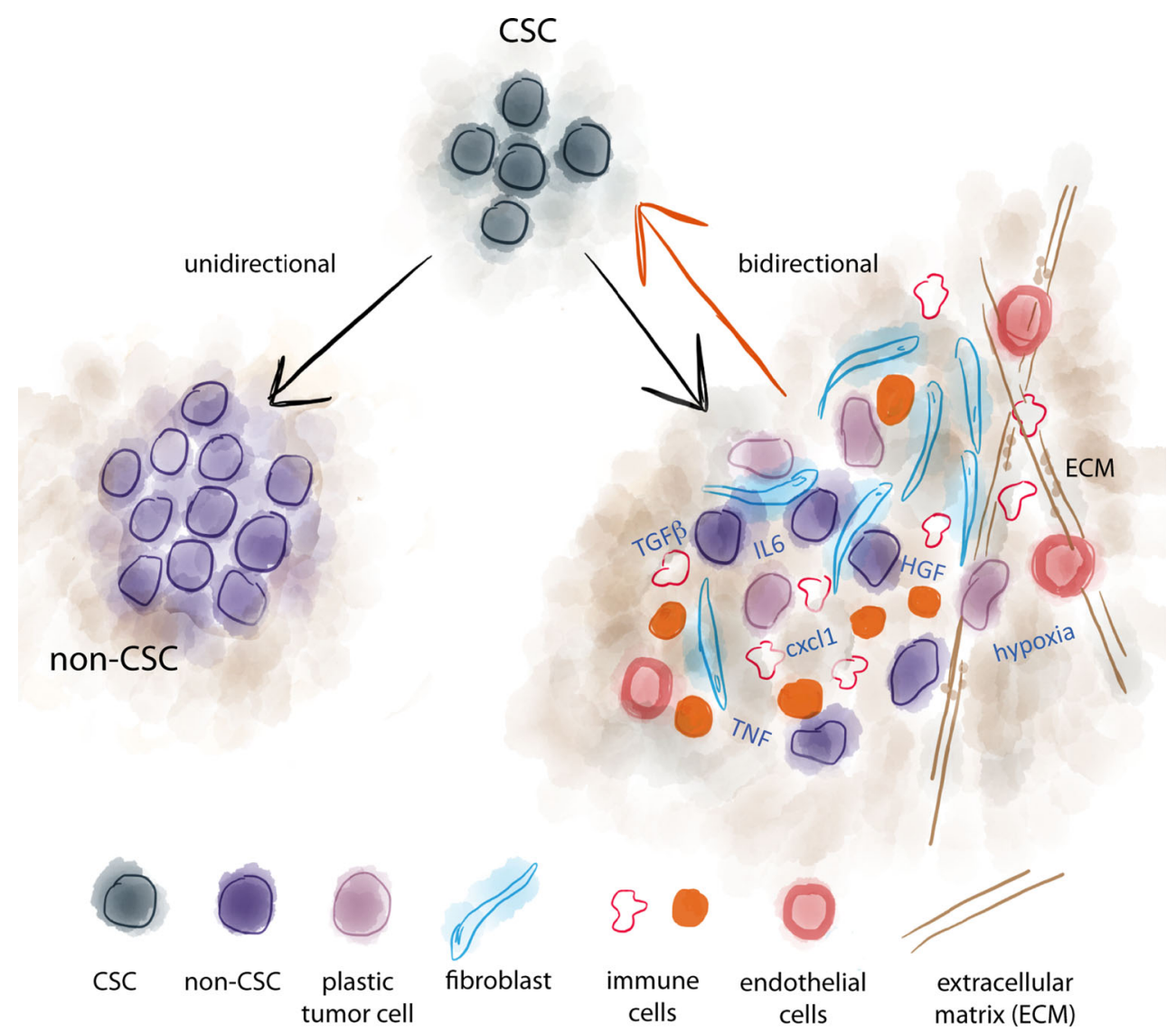

escape the immune system and avoid therapeutic intervention. Several concepts evolved within the last years, suggesting possible causes and sources of tumor heterogeneity and how tumors become metastatic and therapy resistant, finally resulting in tumor relapse and a poor outcome for the patient:

1. Cancer stem cell (CSC) hypothesis: in this unidirectional hierarchical model, only a genetically superior subpopulation of tumor initiating cancer cells can fuel tumor growth by generating more differentiated nonCSC without tumorigenic potential. Accordingly, tumors are subject to a hierarchical organization from the top down, akin to a normal homeostatic tissue hierarchy maintained by normal stem cells [4-6].

2. Stochastic model: Every cell within a tumor or tissue is capable to fuel a tumor [7]. Transformed cells can change their phenotype and their potential by accumulating further mutations, or stochastically change their phenotype reversibly in response to microenvironmental influences. Different populations of subclones with robust properties can become dominant according to a Darwinian evolution process [8-11].

Unidirectional, genetic models of tumorigenesis may only partially explain the source of tumor heterogeneity, as survival in the constantly changing tissue environment rather requires a highly adaptable character. Unpredictable stochastic influences are likely to provoke a reversible, "plastic" process to meet the requirements of rapid adaptation to survive in a dynamic tumor environment.

\section{CSC hypothesis in question}

The CSC hypothesis suggests that there is a small subset of potent tumorigenic cells with stem-cell-like properties that act as the cell of origin to initiate and progress a tumor in a hierarchical fashion. CSC-like cells with the highest carcinogenic potential can undergo symmetric divisions to increase in numbe, $\mathrm{r}$ but majorly perform asymmetric divisions in order to generate more differentiated non-CSC progeny (Fig. 1) [12, 13]. CSCs have the ability to maintain a hierarchical organization of the primary tumor as well as of the corresponding metastatic deposit. Hence, CSC are a minor subpopulation of highly tumorigenic cancer cells possessing the main potential to initiate and progress a tumor alongside with a bulk of their nontumorigenic progeny. The model implies that once a CSC exits the CSC state, it cannot re-enter. However, the proportion of tumorigenic cells following this model is widely 
unclear. Several markers of CSC have been proposed, such as CD24, CD44, CD133, and CD166 for colorectal cancer (CRC) [14-16]. But so far, a robust marker of CSCs has not been identified. The expression of these proposed markers is not strictly confined to, but overlap in, specific groups of stem or progenitor cells, though expression levels may vary. For example, CD166 can be ubiquitously expressed in differentiated murine and human colon epithelial cells [17]. The caveat to the classic CSC model is its simplification towards a rigid, unidirectional nature of tumor development as more and more evidence comes up indicating a rather complex, dynamic and plastic tumor organization, in which tumor cells have the ability to adapt rapidly to multiple different intrinsic and changing extrinsic influences.

There is evidence that neoplastic and normal epithelial cells can re-enter a stem cell state, indicating a dynamic plasticity between CSC and non-CSC populations, depending on various intrinsic but mainly extrinsic factors that confer to non-CSC cells the ability to initiate a tumor de novo, or contribute significantly to tumor aggressiveness. It implies that a subpopulation of tumorigenic cells, constantly changing in phenotype, number and tumor intrinsic location depending on various factors, contributes to tumor progression. Therefore, CSCs act as moving targets, which are difficult to hit therapeutically. Also, the amount of contributing cells remains unpredictable. By means of fate mapping, selective cell ablation, and transplantation assays, given the existence of a permissive environment, it may now be possible to assess the contribution of the different tumor cell populations' potential in a better way. The inability to reproduce the tumorigenic potential of solid human tumor cells with predicted, identified CSC markers undermines the idea of a strict hierarchical organization of tumors. The same tumors of different patients can display an extensively heterogeneous character, implicating either a steep hierarchy of some cells giving rise to large numbers of non-tumorigenic progeny, a shallow hierarchy in which most cells give rise to some non-tumorigenic progeny, or almost no hierarchy, as almost every cell can contribute to tumor growth. Accordingly, the more shallow the hierarchy, the more cells contribute to tumor growth, and hence the worse are the treatment options; e.g., studies in benign and malignant papilloma confirmed a steep hierarchy in benign tumors and a more shallow hierarchy in advanced, untreatable tumors [18]. In primary human CRC, subpopulations of lentivirus-labeled cells [19] behaved different after serial transplantation into mice. Some populations were always abundant and persisted, some were abundant and became rare, and some were rare and became more abundant, suggesting an inherent functional variability apart from genetic variability. In advanced tumors, cancer cells frequently change their phenotype by switching between the mesenchymal and epithelial cell states; e.g. breast cancer cells have even been shown to be more potent in a mesenchymal state than in an epithelial state [20]. So far, reversible transitions between tumorigenic and nontumorigenic states have primarily been shown in vitro, and the actual abundance of interchangeability between different cell states within a tumor and its contribution to tumorigenesis and therapy resistance remains to be addressed in more detail. Therapy resistant cell populations can benefit from the chronic inflammatory tumor microenvironment, as well as from therapy-induced tissue injury, which provides the protecting niche for survived tumor cells, which in turn are predisposed to accumulate further mutations at a higher rate under the selective pressure of the inflammatory conditions [21].

For example, selection for tumor cell subpopulations that harbor genetic amplifications and deletions of genes that are involved in nucleoside synthesis, which target drugs such as methotrexate and 5-fluorouracil [22], can lead to drug resistance. Deep sequencing is one option to assess the composition of subclonal populations; however, it does not capture the potential of reciprocity. Whole genome analyses of somatic mutations demonstrated that only a limited number of driver mutations account for the malignancy of a tumor [23]. Tumors from different tissues show differing genetic variability ranges; e.g., data from AML [24, 25], CLL [26], breast cancer [27, 28] and pancreatic cancer [29, 30] display genetic variabilty to a large extent, while human CRC cells revealed only a small number of genetically variant cells in serially tranplanted cells $[15,16]$.

The instant development of resistance to multiple chemotherapeutics, as well as the reversibility of acquired drug resistance in some patients after exposing them to a second cycle of treatment, indicate that frequently non-mutational events can lead to drug unresponsiveness. Tumor cells respond to microenvironmental conditions by interchanging cell states and changing their phenotype [6]; e.g., quiescent cells could either be in a stem cell-like state or in a highly differentiated state and can resume proliferation once microenvironmental conditions allow it.

This observation challenges the classical, rigid CSC hierarchy model proposing that tumors originate from one fixed subpopulation of cells. The plastic model suggests that a flexible pool of cells within a tissue can revert to or become a tumorigenic cell and vice versa (Fig. 1). Tumor heterogeneity by cell plasticity can be observed independent of the tumor type, indicating coexistence and cooperation of continuous genetic alterations and reversible phenotypic plasticity between differentiated and dedifferentiated stem cell-like states. It is also likely that both CSC as well as a stochastic process apply to human cancers, and that tumors exhibit traits that are generated by both models [31]. 
Inflammatory microenvironment: inflammatory mediators induce phenotypic plasticity

The inflammatory microenvironment is shown to have a crucial role during tumor progression in colon cancer and other tumors, by promoting cell plasticity and therefore heterogeneity. Heterogeneous tumor progression is an adaptive and dynamic process that is guided by soluble factors from the inflammatory stroma in the tumor's adjacency, including fibroblasts, endothelial cells, pluripotent mesenchymal stem cells, as well as diverse immune cells, leading to a change in the tumor's differentiation state, affecting phenotype, cell adhesion, morphology and cytoskeletal dynamics [32-35]. A reciprocal interaction of tumor microenvironment including inflammatory cells, soluble factors and the extracellular matrix (ECM) with tumor cells $[8,36,37]$ leads to dynamic epigenetic and cell signaling network alterations, and ultimately determines the heterogeneity of a tumor (Fig. 1) [38-41].

The inflammatory microenvironment builds up gradually during tumor development. A variety of stromal cells, together with endothelial cells to generate an adequate blood supply for the developing tumor, is conscripted to the site of tumorigenesis. Once present at the tumor site, stromal cells become activated in a mutual crosstalk with tumor cells, altering their normal secretion pattern of cytokines, growth factors, and ECM components to create a "reactive stroma" promoting tumor progression by increasing tumor cell proliferation and degrading basement membranes to enhance invasive ability. For example, microenvironmental TNF- $\alpha$ released from endothelial and stromal cells induces cancer cells to secrete chemokines, attracting myeloid tumor promoting immune cells [42]. Specifically, colon and breast cancer cells are shown to secrete the chemokines Cxcl1 and Cxcl2, leading to the recruitment of $\mathrm{Gr} 1+$ myeloid cells, which in turn secrete S100A8/9, conferring to tumor cells a Stat3- and NF- $\kappa B-$ mediated protective effect against cell death and chemotherapy [43]. Moreover, TNF- $\alpha$ and IL- 6 are shown to force a differentiation state switch to a mesenchymal cell state in epithelial tumor cells, through NF- $\kappa \mathrm{B}$ signal induction in breast, lung, colon tumor cells, as well as melanoma cells [42, 44-47].

The NF- $\mathrm{BB}$ signaling pathway is one of the central signaling pathways involved in the production of the inflammatory cytokines such as IL-6, IL-11, and TNF- $\alpha$, and is therefore responsible for survival, proliferation and cell plasticity in all tumor stages via distinct mechanisms $[48,49]$. NF- $\mathrm{kB}$, activated in myeloid cells, controls the production of inflammatory cytokines, which in turn activate NF- $\mathrm{\kappa B}$ in initiated epithelial cells, leading o transcription of genes that promote cell survival and proliferation and that actively suppress apoptosis [50]. NF- $\kappa \mathrm{B}$ activity also results in the upregulation of inducible nitric oxide synthase (iNOS) in epithelial cells and can lead to DNA damage and hence to cell transformation [51]. Preventing NF- $\mathrm{KB}$ activation in myeloid cells of mice leads to a decreased number and size of colitis-associated tumors [50].

Other microenvironmental components, such as hypoxia and hypoxia-induced VEGF, affect tumor and immune cell plasticity by recruitment of regulatory $\mathrm{T}$ cells and other immune suppressive immune cells contributing to an angiogenic switch [52, 53]. A side effect of hypoxia, altered tumor metabolism and tumor acidification via lactate secretion and anaerobic glycolysis results in an impaired immune effector $\mathrm{T}$ cell function [53] and enhanced recruitment of myeloid derived suppressor cells, further promoting tumor aggressiveness and therapy resistance via microenvironmentally induced tumor plasticity.

The tumor niche also shapes the response to the selective pressure of drug treatment, and may affect the emergence of resistance. Therefore, the composition of the tumor microenvironment crucially impacts patients' survival and relapse after chemotherapy by helping to establish a chemoresistant niche [43]. Increased occurrence of mesenchymal markers corresponds to an increased resistance to chemotherapy and immunotherapy, as has been observed in patients and mouse models [44, 54, 55]. In patients with breast cancer, the presence of macrophages was observed to be indicative of the outcome of therapy success and the survival of tumor cell survival [56]. Therefore, the mutual interaction between microenvironmental components and tumor cells favors increased phenotypic tumor cell and immune cell plasticity, facilitating advanced malignant tumor development. Consequently, the reactive stroma plays a prominent inductive role in the conversion of non-CSC to CSC-like cell states increasing an aggressive tumor character in colon, breast, as well as other cancers [57-60]. The interaction between the inflammatory microenvironment and CSC formation could be demonstrated in a transgenic mouse model of Barrett's esophageal adenocarcinoma [61]. In response to bile acidinduced IL-1 $\beta$ - and IL-6 - dependent inflammation and Dll1-dependent Notch signaling, gastric cardia progenitor cells migrated into the distal esophagus and gave rise to columnar-like metaplasia [62]. Inflammation-induced plasticity of tumor cells and immune cells was also observed in mouse melanoma models [44]. The power of the microenvironment on cell plasticity, apart from cellintrinsic mechanisms, has been detected as well during normal homeostatic lineage differentiation of untransformed intestinal stem cells (ISM), demonstrating that epithelial lineage separation is reversible and therefore not a true lineage commitment. Kim et al. [63] showed that ISC 
are equipotent, and lineage differentiation from ISC does not require differential chromatin priming, as progenitors for different lineages display comparable histone modifications and chromatin access regions, and are rather dependent on environmental stimuli and rely on transcription factor activity (Atoh1) for determining the fate of stem cells or the reversion of differentiated cells into ISM.

\section{Epigenetic changes and tumor heterogeneity}

Mutational alterations are not the only drivers of tumorigenesis and determinants of tumor heterogeneity, as it has become evident that non-mutational and reversible epigenetic events contribute to cell plasticity and significantly impact carcinogenesis. Stochastic epigenetic changes, such as histone and chromatin modifications and altered DNA methylation, occur dynamically in response to environmental signals during and after accumulations of mutations that lead to alterations in the normal cellular development and maintenance program, and hence can result in tumor initiation [64]. Aberrant DNA methylation by upregulation of DNA demethylases occurs soon after APC loss, indicating that APC controls DNA demethylase expression and highlights demethylation as an important event during tumor initiation preceding hyperproliferation [65-67]. Supportingly, Apc ${ }^{\text {min }}$ mice carrying a genetic deletion for the cytidine deaminase Apobec1 exhibit reduced polyp formation [68]. Generally, cancers exhibit a global DNA hypomethylation [69], but in contrast, site-specific gene hypermethylation is also evident. Known global epigenetic events in colorectal cancers and also in breast cancers are hypermethylation of distinct $\mathrm{CpG}$ island shores (CIMP phenotype) and in the promoter regions encoding tumor suppressors such as p16INK4a, cell cycle regulator RB (retinoblastoma protein) or DNA repair enzymes BRCA1 or MLH1 [64, 70, 71]. Besides global changes in DNA methylation and increased trimethylation of $\mathrm{H} 3 \mathrm{~K} 27 \mathrm{me}^{3}$, other posttranslational histone modifications, such as loss of H4K16 acetylation and reduced H4K20 methylation, are common in CRC, and correlate with transcriptional silencing as well as target loci for cancer-specific promoter methylation $[72,73]$. It has been suggested that epigenetics contribute to intestinal cell fate determination, which is frequently blocked during tumorigenesis. During tumor initiation after the loss of APC, epigenetic changes are shown to precede hyperproliferation and even to activate oncogenes such as Ras [74]. As a number of studies have failed to correlate APC loss with increased Wnt activity through increased $\beta$-catenin nuclear translocation, epigenetic events are likely to assist in adenoma development. Epigenetic alterations are shown to be responsible for increased expression of drug transporters, DNA-repair enzymes and pro-apoptotic factors that protect against cytotoxic drugs during tumor progression, causing rapid disease relapses $[38,75]$.

\section{Cell plasticity and reciprocity in different tumors}

The reversible character and dedifferentiation of tumor cells is a plastic process described by the loss of lineagespecific cell marker expression and the reinitiation of a stem-cell-like expression pattern. The degree of dedifferentiation is highly context and tumor dependent, but is likely to occur throughout all stages of tumorigenesis. Normal, differentiated epithelia are able to convert into CSCs; e.g., the inflammatory microenvironment in inflammatory bowel disease patients may predispose inflamed epithelial cells towards transformation [59]. In advanced tumors, the inflammatory microenvironment could allow the dedifferentiation of more differentiated tumor cells into CSCs to replenish therapeutically eliminated tumor stem cells. Among all reported incidents of cell type plasticity within tumors, melanoma tumors are reported to belong to the most plastic tumor types with a high tumor initiating frequency [76]. Recent reports demonstrated that melanoma cells, sorted into specific cellular subpopulations on the basis of the expression of surface markers, spontaneously re-establish phenotypic heterogeneity by switching between a highly proliferative melanocytic state and a less differentiated invasive state in vitro. Serial transplantation experiments revealed that melanoma cells exist in a dynamic equilibrium between differentiated and dedifferentiated subpopulations that express or lack the melanocytic signature in vivo, respectively [77]. TNF- $\alpha$ could reversibly switch both mouse and human melanoma cells from a melanocytic to a non-melanocytic phenotype, and thereby selectively impair the recognition of specific melanocytic antigens by cytotoxic T-cells (CTLs) [44, 78, 79]. The adaption to therapy-induced inflammatory signals in the environment in a remarkable short time period indicates the involvement of altered epigenetic states in cell plasticity. For example, melanoma cells expressing the histone demethylase JARID1B are required for tumor growth, which spontaneously can arise from JARID1B negative melanoma cells [80]. Furthermore, Sharma et al. demonstrated that a small subpopulation of non-small cell lung carcinoma cells marked by JARID1A existed in a drug-tolerant state. Importantly, cells could exit that state and again re-enter it later on [81]. These studies reveal that cell plasticity can lead to a drug tolerant state, implicating substantial challenges for effective anti-tumor therapeutics. Inflammation induced reversibility between an undifferentiated stem-cell-like state or a CSC-like tumor initiating phenotype and a differentiated state has also been 
demonstrated in breast cancer and colon cancer mouse models [45-47, 59]. Breast cancer cells stochastically switch between different cell states resembling their physiological mammary gland epithelium [57]. In addition to the expression of EMT factors, non-CSC CD441 ${ }^{\text {ow }} \mathrm{C}$ D2 $4^{\text {low }}$ breast cancer cells were able to acquire a tumor initiating $\mathrm{CD} 44^{\mathrm{hi}} \mathrm{CD} 24^{\mathrm{lo}}$ cell surface marker profile characteristic of breast CSC in vivo without genetic manipulation [20, 57, 82]. Mechanistically, Chaffer et al. [41] gave proof that plastic breast non-CSC maintain the ZEB1 promoter in a bivalent chromatin configuration, enabling them to switch rapidly to an active chromatin state in response to environmental stimuli such as TGF $\beta$.

In the intestinal epithelium, high cell plasticity can also be observed throughout the normal homeostatic and differentiation process. Intestinal stem cells, marked by Lgr5+, are located at the bottom of the crypt and interact with the surrounding microenvironmental niche to maintain normal epithelial differentiation and stem cells in the crypt. Recent studies demonstrate that according to the neutral drift model, equipotent crypt Lgr5+ stem cells divide symmetrically in the crypt; however, their fate is determined by the surrounding niche [83]. Once stem cells leave the crypt by losing contact with adjacent Paneth cells, they start migrating upwards along the epithelium and differentiate into progenitors and epithelial cells. In case of an injury, affected Lgr5+ stem cells are shown to be replenished by either Dll1+ secretory progenitors or by Paneth cell precursors, which both have the ability to revert to Lgr5+ expressing cells [84, 85].

In the context of intestinal tumorigenesis, it has been demonstrated based on a mouse model that TNF- $\alpha$ dependent NF- $\kappa$ B activation amplified Wnt activity in transformed epithelial cells, leading to re-expression of a stem cell transcription pattern conferring not only Lgr5+ ISM, but also Lgr5- epithelial cells the potential to reexpress Lgr5+ and convert to a stem cell [59]. Lgr5+ as well as Lgr5- cells gave rise to tumors and even formed spheroids in culture, supporting the unidirectional hierarchical CSC theory only to a limited extent, but rather emphasizing an inflammation-directed bidirectional conversion process in the context of Wnt-dependent tumorigenesis. By use of a multicolor reporter mouse that activates Cre recombinase in Lgr5+ cells, it could be shown that Lgr5+ cells in the normal epithelium give rise to adenomas, and after another recombination pulse, a color switch in Lgr5+ cells indicated that Lgr5+ cells within adenomas still contribute majorly to tumor growth [86]. As most of the Lgr5+ progeny were Lgr5-, it can be speculated that most Lgr5+ cells spawn fewer or not very proliferative Lgr5- cells; however, as Lgr5- cells have not yet been traced within adenoma and tumor formation, their actual contribution in this context compared to Lgr5+ cells is still elusive. It will be interesting to test whether adenomas that do indeed exhibit a hierarchical organization during tumor initiation continue to do so after progressing to malignancy. In summary, during homeostasis, ISM and progenitors are dynamically reversible, like non-CSC and CSC during carcinogenesis, and this is dependent on the niche, which corroborates the role of the microenvironment in cell fate determination during tissue homeostasis, and also during tumor development.

The impact of cell plasticity on phenotypic and functional heterogeneity is also crucial in the context of its contribution to epithelial-mesenchymal transition (EMT) during tumor progression. EMT is an early developmental process, naturally occurring during gastrulation and neural crest formation, as well as for tissue homeostasis [87]. During tumor progression, this embryonic EMT program is reinitiated, leading to changes in cell function, morphology and phenotype, and initiates invasive potential and cell mobility by the re-emergence of stem cell characteristics, which has been documented in colon cancer and other tumors [87-89]. The fact that preferably cells at the invasive tumor front exposed to an inflammatory microenvironment acquire CSC characteristics in response to the stromal signals, reinforces the assumption of dedifferentiation as stochastic, dynamic and bidirectional process in response to extrinsic factors rather than solely induced via intrinsic oncogenic transformation. During EMT, stroma derived factors, including Wnt, HGF, TGF- $\beta$, FGF, and EGF, as well as a number of cytokines, lead to the activation of transcriptional repressors, including ZEB1, Twist, Snail1 and 2, which directly and indirectly inhibit E-cadherin transcription [54, 88, 90, 91]. In a mouse model developing highly invasive colorectal tumors, it could be shown that tumor cells at the invasive front performed EMT by upregulating EMT markers such as Twist 1 and Snaill, which conversely downregulated epithelial E-Cadherin in a NF- $\kappa B$-dependent manner. The recruitment of inflammatory myeloid cells further accelerated the EMT process and supported tumor cell survival through additional Stat3 activation in tumor cells [42]. Spatiotemporal plasticity and reciprocity are essential in both tumor cells and the microenvironment during tumor progression, and are subject to further drift, with cells moving from one environment to another. The concept of dynamic reciprocity was originally termed in the context of wound healing by Paul Bornstein [93], and was expanded to the context of cancer in 2008 [93, 94]. Accordingly, plasticity and reciprocity account for the morphologic and functional intertumoral and intratumoral heterogeneity driven by various mentioned mechanisms in response to hypoxia and severe metabolic stress [95], as well as chronic growth factor stimulation and inflammation [96, 97], in order to adapt to altering environmental conditions. The change of 
cell position is also critical for the induction of plasticity and reciprocity. EMT gives access to different physical environments and molecular structures at the primary tumor site, as well as at the metastatic site. Consequently, invasion and metastasis are both cause and consequence of plasticity and reciprocity. In terms of colorectal carcinogenesis the change in cell position is given through a high turnover pace and movement of dividing and differentiating epithelial cells out of the crypt upwards, and therefore exposure to a changing microenvironment, which may facilitate cell plasticity of predisposed cells of all differentiation states to initiate tumorigenesis.

\section{Therapeutic challenges}

The phenotypic changes associated with a switch between various cell differentiation states within a tumor account for differing responsiveness to several chemotherapeutics [98]. Therefore, cell plasticity is a key determinant of drug resistance in cancer cells. The role of the inflammatory microenvironment as a major trigger for reversible, stochastic cell state transitions, and therefore facilitating tumor relapse, has been widely underestimated thus far [43, 99]. However, targeting the tumor environment to restrict cell plasticity rather than merely targeting subpopulations of tumor cells opens a new avenue for promising treatment options. In a mouse model of glioblastoma, bone-marrowderived $\mathrm{CD} 11 \mathrm{~b}+$ myeloid cells contributed to tumor regrowth by promoting a functional tumor vasculature following radiotherapy, which could be inhibited by pharmacological blockade of SDF1-binding to its receptor CXCR4 [100]. Moreover, therapeutic blockade of macrophage recruitment in breast cancer patients increased the survival chance after therapy [43, 99]. Resistance against immune therapy accounts for the cancer immunoediting theory in which cancer and immune cell co-evolution are liable to the Darwinian selection of therapy resistant tumor cell variants that escape cytotoxic $\mathrm{T}$ cells either through downregulation of $\mathrm{T}$ cell antigens or MHC molecules, but primarily also through an effector T-cell suppressive inflammatory microenvironment, as has been demonstrated in transgenic mouse models of melanoma [101-103]. To what extent cell plasticity contributes to tumor malignancy is still unknown, but new treatment approaches targeting inflammation induced cell plasticity rather than single subpopulations of cells are in demand in order to prevent resistance to immunotherapies and chemotherapies. However, diagnosing the degree of plasticity and cell state dynamics in order to assess the predicted tumor malignancy will be challenging compared to genetic alterations. Cell plasticity is hard to track, due to its transient character and high reversibility depending on the surrounding conditions, and cannot be captured in a representative way by the snapshot of a classic tumor biopsy and subsequent histological analysis. One approach to evaluate cell state dynamics and plasticity may be in vivo non-invasive molecular imaging, as hypoxia, apoptosis, acidosis, cell signaling and inflammation can be considered, which are all important factors accounting for a high plasticity $[104,105]$.

Prediction of the transplantation and therapy response depend on the form and extent of cell type plasticity, as the differentiation of tumorigenic cells into non-tumorigenic progeny can either be irreversible, partially reversible or readily reversible, of which a fully reversible cell is most difficult to erase. Yet, therapies forcing CSC to differentiate to minimize CSC populations, as well as intervention of their proliferation, may provide at least one strategy to target CSC populations. Tumorigenic CSC of some cancers are intrinsically resistant to certain therapies, such as some glioblastoma or certain types of breast cancer cells, in which tumorigenic cells were found to be enriched after irradiation [106, 107]. Similar observations were made in cyclophosphamide-treated CRC cells [108]. However, it may not be generally true that CSC are defined by therapy resistance, but instead they may be more susceptible to other therapy methods than non-tumorigenic cells. To achieve a high therapeutic efficacy rate, interdisciplinary approaches are required to target tumor plasticity, e.g., inhibiting signaling in the microenvironment in addition to immunotherapy and genotoxic therapies, as well as using radiotherapy and oncogenic signaling inhibitors combined with $\mathrm{T}$ cell directed immunotherapy.

\section{Outlook}

An emerging huge body of work is supporting a dynamic model of tumorigenesis in which interconversions between low and high tumorigenic states occur frequently, thereby increasing tumorigenic and malignant potential. Therefore, a deeper investigation of genetic heterogeneity and cell plasticity will be required to achieve a better understanding of the tumorigenic potential of tumors and how to target tumorigenic subpopulations, or rather, prevent cell plasticity and the emergence of highly tumorigenic cells. So far, the question of to what extent tumor plasticity contributes to tumor phenotypic and functional heterogeneity is still unaddressed. In some cancers, tumorigenic cells are rare with a clear hierarchy; in others, they are more abundant with a less obvious hierarchy; there may also be tumors with coexistence of tumorigenic cells with different potential in which tumors follow the CSC model only partially, which makes it difficult to determine the actual tumorigenic potential. Another difficulty in determining the amount of tumorigenic cells is the lack of valid 
combination of CSC markers available to isolate a CSC population with a high degree of purity, so a substantial amount of patient samples needs to be screened to identify a more reliable set of CSC markers to analyze the heterogeneity among patients. A combination of immunotherapy/ chemotherapy and inhibitors of oncogenic signaling is likely to be most beneficial for a good outcome, and the key may be the right time schedule of application to achieve best results regarding antigenic landscape and oncogenic signaling cascade variations. Supported by hitherto findings and regarding the complexity of tumor development as well as the variations among different cancer types or the variations among patients with the same cancer type, the different tumor heterogeneity source concepts are likely to coexist and do not exclude each other, but may altogether provide better explanations to the heterogeneity within tumors and among tumors, than only referring to one concept. A combination of extrinsic signaling and cell-intrinsic genetic and epigenetic variations facilitate the event of dynamic interconversion between non-CSC and CSC cell states, questioning the existence of a rigid CSC hierarchy and rather supporting a transient, dynamic, short-lives hierarchical monopoly of some CSClike cells, which may switch into more differentiated cell states; however, they can be easily replaced by non-CSC populations acquiring CSC expression patterns. The generation of tumor-initiating and metastatic CSC from nonCSCs implicates that tumorigenesis is a far more complex process than can be expected according to the classic CSC model, as all types of cells independent of their differentiated state are capable of switching to a tumor promoting cell state de novo. Hence, the pool of tumorigenic cells is not only limited to stem cells or a subpopulation of cells, but is larger than expected and dynamic depending on extrinsic and intrinsic factors; hence calling for new individual therapeutic strategies. The dynamic cell plasticity concept for tumor development suggests that the cells responsible for aggressive tumorigenesis are moving targets, and only combined therapies inhibiting CSC proliferation or forcing CSCs to exit the CSC-state, as well as prevention of cell state switching, may provide the most effective therapeutic system to improve patient survival.

According to the traditional, hierarchical CSC model, CSCs are on top of the hierarchy and fuel tumor growth in a unidirectional top-down manner by spawning a bulk of more differentiated non-CSC, which cannot re-enter the CSC state again. The plastic, bidirectional CSC model suggests that non-CSC tumor cells can rapidly change their phenotype and possibly revert back towards a CSC state in response to changes in the niche, depending on hypoxia, $\mathrm{pH}$ changes, structural tissue remodeling and infiltration of inflammatory cells secreting pro-inflammatory mediators such as cxcl1, IL-6, TNF- $\alpha$, HGF or TGF- $\beta$.
Acknowledgments The author is supported by a postdoctoral fellowship from EMBO.

Conflict of interest The authors declare that they have no conflict of interest.

\section{References}

1. Broadbridge VT, et al. Do metastatic colorectal cancer patients who present with late relapse after curative surgery have a better survival? Br J Cancer. 2013;109(5):1338-43.

2. Honeth G, et al. The CD44 +/CD24- phenotype is enriched in basal-like breast tumors. Breast Cancer Res. 2008;10(3):R53.

3. Stoecklein $\mathrm{NH}$, et al. Direct genetic analysis of single disseminated cancer cells for prediction of outcome and therapy selection in esophageal cancer. Cancer Cell. 2008;13(5):441-53.

4. Magee JA, Piskounova E, Morrison SJ. Cancer stem cells: impact, heterogeneity, and uncertainty. Cancer Cell. 2012; 21(3):283-96.

5. Clevers H. The cancer stem cell: premises, promises and challenges. Nat Med. 2011;17(3):313-9.

6. Frank NY, Schatton T, Frank MH. The therapeutic promise of the cancer stem cell concept. J Clin Invest. 2010;120(1):41-50.

7. Bakhoum SF, Compton DA. Chromosomal instability and cancer: a complex relationship with therapeutic potential. J Clin Invest. 2012;122(4):1138-43.

8. Marusyk A, Almendro V, Polyak K. Intra-tumour heterogeneity: a looking glass for cancer? Nat Rev Cancer. 2012;12(5):323-34.

9. Gerlinger M, Swanton C. How Darwinian models inform therapeutic failure initiated by clonal heterogeneity in cancer medicine. Br J Cancer. 2010;103(8):1139-43.

10. Swanton C. Intratumor heterogeneity: evolution through space and time. Cancer Res. 2012;72(19):4875-82.

11. Gillies RJ, Verduzco D, Gatenby RA. Evolutionary dynamics of carcinogenesis and why targeted therapy does not work. Nat Rev Cancer. 2012;12(7):487-93.

12. Visvader JE, Lindeman GJ. Cancer stem cells: current status and evolving complexities. Cell Stem Cell. 2012;10(6):717-28.

13. Bonnet D, Dick JE. Human acute myeloid leukemia is organized as a hierarchy that originates from a primitive hematopoietic cell. Nat Med. 1997;3(7):730-7.

14. Dalerba P, et al. Phenotypic characterization of human colorectal cancer stem cells. Proc Natl Acad Sci USA. 2007;104(24):10158-63.

15. O'Brien CA, et al. A human colon cancer cell capable of initiating tumour growth in immunodeficient mice. Nature. 2007;445(7123):106-10.

16. Ricci-Vitiani L, et al. Identification and expansion of human colon-cancer-initiating cells. Nature. 2007;445(7123):111-5.

17. Shmelkov SV, et al. CD133 expression is not restricted to stem cells, and both CD133 + and CD133- metastatic colon cancer cells initiate tumors. J Clin Invest. 2008;118(6):2111-20.

18. Perez-Losada J, Balmain A. Stem-cell hierarchy in skin cancer. Nat Rev Cancer. 2003;3(6):434-43.

19. Kreso A, et al. Variable clonal repopulation dynamics influence chemotherapy response in colorectal cancer. Science. 2013;339(6119):543-8.

20. Mani SA, et al. The epithelial-mesenchymal transition generates cells with properties of stem cells. Cell. 2008;133(4):704-15.

21. Colotta F, et al. Cancer-related inflammation, the seventh hallmark of cancer: links to genetic instability. Carcinogenesis. 2009;30(7):1073-81.

22. Wang TL, et al. Digital karyotyping identifies thymidylate synthase amplification as a mechanism of resistance to 
5-fluorouracil in metastatic colorectal cancer patients. Proc Natl Acad Sci USA. 2004;101(9):3089-94.

23. Salk JJ, Fox EJ, Loeb LA. Mutational heterogeneity in human cancers: origin and consequences. Annu Rev Pathol. 2010;5:51-75.

24. Ding L, et al. Clonal evolution in relapsed acute myeloid leukaemia revealed by whole-genome sequencing. Nature. 2012;481(7382):506-10.

25. Walter MJ, et al. Clonal architecture of secondary acute myeloid leukemia. N Engl J Med. 2012;366(12):1090-8.

26. Schuh A, et al. Monitoring chronic lymphocytic leukemia progression by whole genome sequencing reveals heterogeneous clonal evolution patterns. Blood. 2012;120(20):4191-6.

27. Navin $\mathrm{N}$, et al. Tumour evolution inferred by single-cell sequencing. Nature. 2011;472(7341):90-4.

28. Nik-Zainal S, et al. The life history of 21 breast cancers. Cell. 2012;149(5):994-1007.

29. Jones $\mathrm{S}$, et al. Core signaling pathways in human pancreatic cancers revealed by global genomic analyses. Science. 2008;321(5897):1801-6.

30. Yachida S, et al. Distant metastasis occurs late during the genetic evolution of pancreatic cancer. Nature. 2010; 467(7319):1114-7.

31. Shackleton M, et al. Heterogeneity in cancer: cancer stem cells versus clonal evolution. Cell. 2009;138(5):822-9.

32. Egeblad M, Rasch MG, Weaver VM. Dynamic interplay between the collagen scaffold and tumor evolution. Curr Opin Cell Biol. 2010;22(5):697-706.

33. Picchio M, et al. Intratumoral spatial distribution of hypoxia and angiogenesis assessed by 18F-FAZA and 125I-Gluco-RGD autoradiography. J Nucl Med. 2008;49(4):597-605.

34. Friedl P, Wolf K. Plasticity of cell migration: a multiscale tuning model. J Cell Biol. 2010;188(1):11-9.

35. Sanz-Moreno V, Marshall CJ. The plasticity of cytoskeletal dynamics underlying neoplastic cell migration. Curr Opin Cell Biol. 2010;22(5):690-6.

36. Hanahan D, Coussens LM. Accessories to the crime: functions of cells recruited to the tumor microenvironment. Cancer Cell. 2012;21(3):309-22.

37. Junttila MR, de Sauvage FJ. Influence of tumour micro-environment heterogeneity on therapeutic response. Nature. 2013;501(7467):346-54.

38. Wilting RH, Dannenberg JH. Epigenetic mechanisms in tumorigenesis, tumor cell heterogeneity and drug resistance. Drug Resist Updat. 2012;15(1-2):21-38.

39. Singh A, Settleman J. EMT, cancer stem cells and drug resistance: an emerging axis of evil in the war on cancer. Oncogene. 2010;29(34):4741-51.

40. Baylin SB. Resistance, epigenetics and the cancer ecosystem. Nat Med. 2011;17(3):288-9.

41. Chaffer CL, et al. Poised chromatin at the ZEB1 promoter enables breast cancer cell plasticity and enhances tumorigenicity. Cell. 2013;154(1):61-74.

42. Schwitalla S, et al. Loss of p53 in enterocytes generates an inflammatory microenvironment enabling invasion and lymph node metastasis of carcinogen-induced colorectal tumors. Cancer Cell. 2013;23(1):93-106.

43. Acharyya S, et al. A CXCL1 paracrine network links cancer chemoresistance and metastasis. Cell. 2012;150(1):165-78.

44. Landsberg J, et al. Melanomas resist T-cell therapy through inflammation-induced reversible dedifferentiation. Nature. 2012;490(7420):412-6.

45. Asiedu MK, et al. TGFbeta/TNF(alpha)-mediated epithelialmesenchymal transition generates breast cancer stem cells with a claudin-low phenotype. Cancer Res. 2011;71(13):4707-19.
46. Knutson KL, et al. Immunoediting of cancers may lead to epithelial to mesenchymal transition. J Immunol. 2006;177(3): 1526-33.

47. Santisteban M, et al. Immune-induced epithelial to mesenchymal transition in vivo generates breast cancer stem cells. Cancer Res. 2009;69(7):2887-95.

48. Oeckinghaus A, Hayden MS, Ghosh S. Crosstalk in NF-kappaB signaling pathways. Nat Immunol. 2011;12(8):695-708.

49. Grivennikov SI. Inflammation and colorectal cancer: colitisassociated neoplasia. Semin Immunopathol. 2013;35(2):229-44.

50. Greten FR, et al. IKKbeta links inflammation and tumorigenesis in a mouse model of colitis-associated cancer. Cell. 2004;118(3):285-96.

51. Shaked H, et al. Chronic epithelial NF-kappaB activation accelerates APC loss and intestinal tumor initiation through iNOS up-regulation. Proc Natl Acad Sci USA. 2012;109(35): 14007-12.

52. Motz GT, Coukos G. The parallel lives of angiogenesis and immunosuppression: cancer and other tales. Nat Rev Immunol. 2011;11(10):702-11.

53. Hansen W, et al. Neuropilin 1 deficiency on CD4+ Foxp3 + regulatory $\mathrm{T}$ cells impairs mouse melanoma growth. J Exp Med. 2012;209(11):2001-16.

54. Yao Z, et al. TGF-beta IL-6 axis mediates selective and adaptive mechanisms of resistance to molecular targeted therapy in lung cancer. Proc Natl Acad Sci USA. 2010;107(35):15535-40.

55. Creighton CJ, et al. Residual breast cancers after conventional therapy display mesenchymal as well as tumor-initiating features. Proc Natl Acad Sci USA. 2009;106(33):13820-5.

56. DeNardo DG, et al. Leukocyte complexity predicts breast cancer survival and functionally regulates response to chemotherapy. Cancer Discov. 2011;1(1):54-67.

57. Chaffer CL, et al. Normal and neoplastic nonstem cells can spontaneously convert to a stem-like state. Proc Natl Acad Sci USA. 2011;108(19):7950-5.

58 . Vermeulen L, et al. Wnt activity defines colon cancer stem cells and is regulated by the microenvironment. Nat Cell Biol. 2010;12(5):468-76.

59. Schwitalla $S$, et al. Intestinal tumorigenesis initiated by dedifferentiation and acquisition of stem-cell-like properties. Cell. 2013;152(1-2):25-38.

60. Scheel $\mathrm{C}$, et al. Paracrine and autocrine signals induce and maintain mesenchymal and stem cell states in the breast. Cell. 2011;145(6):926-40.

61. Quante M, et al. Bile acid and inflammation activate gastric cardia stem cells in a mouse model of Barrett-like metaplasia. Cancer Cell. 2012;21(1):36-51.

62. Quante M, et al. Barrett esophagus: what a mouse model can teach us about human disease. Cell Cycle. 2012;11(23): 4328-38.

63. Kim $\mathrm{TH}$, et al. Broadly permissive intestinal chromatin underlies lateral inhibition and cell plasticity. Nature. 2014. doi:10. 1038/nature12903

64. Berdasco M, Esteller M. Aberrant epigenetic landscape in cancer: how cellular identity goes awry. Dev Cell. 2010;19(5):698-711.

65. Rai K, et al. DNA demethylation in zebrafish involves the coupling of a deaminase, a glycosylase, and gadd45. Cell. 2008;135(7):1201-12.

66. Rai K, et al. DNA demethylase activity maintains intestinal cells in an undifferentiated state following loss of APC. Cell. 2010;142(6):930-42.

67. Nadauld LD, et al. Adenomatous polyposis coli control of retinoic acid biosynthesis is critical for zebrafish intestinal development and differentiation. J Biol Chem. 2004;279(49):51581-9. 
68. Blanc V, et al. Deletion of the AU-rich RNA binding protein Apobec-1 reduces intestinal tumor burden in $\operatorname{Apc}(\min )$ mice. Cancer Res. 2007;67(18):8565-73.

69. Feinberg AP, Vogelstein B. Hypomethylation distinguishes genes of some human cancers from their normal counterparts. Nature. 1983;301(5895):89-92.

70. Jones PA, Baylin SB. The epigenomics of cancer. Cell. 2007;128(4):683-92.

71. Esteller M. Epigenetics provides a new generation of oncogenes and tumour-suppressor genes. Br J Cancer. 2007;96(Suppl): R26-30.

72. Fraga MF, et al. Loss of acetylation at Lys16 and trimethylation at Lys20 of histone $\mathrm{H} 4$ is a common hallmark of human cancer. Nat Genet. 2005;37(4):391-400.

73. van Roon EH, et al. BRAF mutation-specific promoter methylation of FOX genes in colorectal cancer. Clin Epigenetics. 2013;5(1):2.

74. Baylin SB, Ohm JE. Epigenetic gene silencing in cancer-a mechanism for early oncogenic pathway addiction? Nat Rev Cancer. 2006;6(2):107-16.

75. Huang S, et al. MED12 controls the response to multiple cancer drugs through regulation of TGF-beta receptor signaling. Cell. 2012;151(5):937-50.

76. Quintana E, et al. Efficient tumour formation by single human melanoma cells. Nature. 2008;456(7222):593-8.

77. Hoek KS, et al. In vivo switching of human melanoma cells between proliferative and invasive states. Cancer Res. 2008;68(3):650-6.

78. Quintana E, et al. Phenotypic heterogeneity among tumorigenic melanoma cells from patients that is reversible and not hierarchically organized. Cancer Cell. 2010;18(5):510-23.

79. Boiko AD, et al. Human melanoma-initiating cells express neural crest nerve growth factor receptor CD271. Nature. 2010;466(7302):133-7.

80. Roesch A, et al. A temporarily distinct subpopulation of slowcycling melanoma cells is required for continuous tumor growth. Cell. 2010;141(4):583-94.

81. Sharma SV, et al. A chromatin-mediated reversible drug-tolerant state in cancer cell subpopulations. Cell. 2010;141(1):69-80.

82. Morel AP, et al. Generation of breast cancer stem cells through epithelial-mesenchymal transition. PLoS One. 2008;3(8):e2888.

83. Lopez-Garcia $\mathrm{C}$, et al. Intestinal stem cell replacement follows a pattern of neutral drift. Science. 2010;330(6005):822-5.

84. van Es JH, et al. Dll1 + secretory progenitor cells revert to stem cells upon crypt damage. Nat Cell Biol. 2012;14(10):1099-104.

85. Buczacki SJ, et al. Intestinal label-retaining cells are secretory precursors expressing Lgr5. Nature. 2013;495(7439):65-9.

86. Schepers AG, et al. Lineage tracing reveals Lgr5 + stem cell activity in mouse intestinal adenomas. Science. 2012;337(6095):730-5.

87. Thiery JP, et al. Epithelial-mesenchymal transitions in development and disease. Cell. 2009;139(5):871-90.

88. Spaderna S, et al. A transient, EMT-linked loss of basement membranes indicates metastasis and poor survival in colorectal cancer. Gastroenterology. 2006;131(3):830-40.

89. Prall F, Weirich V, Ostwald C. Phenotypes of invasion in sporadic colorectal carcinomas related to aberrations of the adenomatous polyposis coli (APC) gene. Histopathology. 2007;50(3):318-30
90. Li G, et al. Downregulation of E-cadherin and Desmoglein 1 by autocrine hepatocyte growth factor during melanoma development. Oncogene. 2001;20(56):8125-35.

91. Toh B, et al. Mesenchymal transition and dissemination of cancer cells is driven by myeloid-derived suppressor cells infiltrating the primary tumor. PLoS Biol. 2011;9(9):e1001162.

92. Bornstein P, McPherson J, Sage EH. Synthesis and secretion of structural macromolecules by endothelial cells in culture. In: Nossel HL, Vogel HJ, editors. Pathobiology of the endothelial cell, P\&S biomedical sciences symposia, vol 6, 1982. pp. 215228

93. Nelson CM, et al. Change in cell shape is required for matrix metalloproteinase-induced epithelial-mesenchymal transition of mammary epithelial cells. J Cell Biochem. 2008;105(1):25-33.

94. Xu R, Boudreau A, Bissell MJ. Tissue architecture and function: dynamic reciprocity via extra- and intra-cellular matrices. Cancer Metastasis Rev. 2009;28(1-2):167-76.

95. Bertout JA, Patel SA, Simon MC. The impact of O2 availability on human cancer. Nat Rev Cancer. 2008;8(12):967-75.

96. Allinen $\mathrm{M}$, et al. Molecular characterization of the tumor microenvironment in breast cancer. Cancer Cell. 2004;6(1): 17-32.

97. Polyak K, Haviv I, Campbell IG. Co-evolution of tumor cells and their microenvironment. Trends Genet. 2009;25(1):30-8.

98. Gupta PB, et al. Stochastic state transitions give rise to phenotypic equilibrium in populations of cancer cells. Cell. 2011;146(4):633-44.

99. Nakasone ES, et al. Imaging tumor-stroma interactions during chemotherapy reveals contributions of the microenvironment to resistance. Cancer Cell. 2012;21(4):488-503.

100. Kioi M, et al. Inhibition of vasculogenesis, but not angiogenesis, prevents the recurrence of glioblastoma after irradiation in mice. J Clin Invest. 2010;120(3):694-705.

101. Schreiber RD, Old LJ, Smyth MJ. Cancer immunoediting: integrating immunity's roles in cancer suppression and promotion. Science. 2011;331(6024):1565-70.

102. Meyer C, et al. Chronic inflammation promotes myeloid-derived suppressor cell activation blocking antitumor immunity in transgenic mouse melanoma model. Proc Natl Acad Sci USA. 2011;108(41):17111-6.

103. Soudja SM, et al. Tumor-initiated inflammation overrides protective adaptive immunity in an induced melanoma model in mice. Cancer Res. 2010;70(9):3515-25.

104. Brogan J, et al. Imaging molecular pathways: reporter genes. Radiat Res. 2012;177(4):508-13.

105. Glunde K, Bhujwalla ZM. Metabolic tumor imaging using magnetic resonance spectroscopy. Semin Oncol. 2011;38(1): 26-41.

106. Lagadec C, et al. Survival and self-renewing capacity of breast cancer initiating cells during fractionated radiation treatment. Breast Cancer Res. 2010;12(1):R13.

107. Bao S, et al. Glioma stem cells promote radioresistance by preferential activation of the DNA damage response. Nature. 2006;444(7120):756-60.

108. Dylla SJ, et al. Colorectal cancer stem cells are enriched in xenogeneic tumors following chemotherapy. PLoS One. 2008;3(6):e2428. 